\title{
Reverse Takotsubo cardiomyopathy: a comprehensive review
}

\author{
Hamza H. Awad ${ }^{1}$, Ashley R. McNeal ${ }^{2}$, Hemant Goyal ${ }^{3}$ \\ ${ }^{1}$ Department of Community Medicine/Internal Medicine, ${ }^{2}$ Medical Student, ${ }^{3}$ Department of Internal Medicine, Mercer University School of \\ Medicine, Macon, GA, USA \\ Contributions: (I) Conception and design: HH Awad, H Goyal; (II) Administrative support: None; (III) Provision of study materials or patients: HH \\ Awad, AR McNeal; (IV) Collection and assembly of data: HH Awad, AR McNeal; (V) Data analysis and interpretation: All authors; (VI) Manuscript \\ writing: All authors; (VII) Final approval of manuscript: All authors. \\ Correspondence to: Hemant Goyal, MD, FACP. Assistant Professor of Medicine, Mercer University School of Medicine, Macon, GA 31201, USA. \\ Email: goyal_h@mercer.edu.
}

\begin{abstract}
Takotsubo cardiomyopathy (TTC) was first described in Japan in the 1980s. It is described as an acute but often reversible left ventricular (LV) dysfunction mainly triggered by emotional or physical stress. Multiple variants of TTC have been reported including reverse Takotsubo cardiomyopathy (rTTC) which is a variant characterized by the basal akinesis/hypokinesis associated with apical hyperkinesis that resolves spontaneously. The hallmark of rTTC is a clinical presentation similar to an acute coronary syndrome (ACS), with no evident obstructive coronary artery disease. The incidence of TTC is estimated to be $2 \%$ of all troponin-positive patients presenting with suspected ACS. The proportion of patients presenting with the rTTC variant out of all TTC patients in published literature has been variable, ranging from 1-23\%. Reverse Takotsubo has been associated with younger age, less decrease in left ventricular ejection fraction (LVEF), and more neurological disease compared to the TTC. While the exact mechanism of rTTC is unknown, hypothesized mechanisms include catecholamine cardiotoxicity, coronary artery spasm, coronary microvasculature impairment, and estrogen deficiency. Patients with rTTC typically present with chest pain and/or dyspnea after an emotional or physically stressful event. rTTC can also be triggered by intracranial hemorrhage, general anesthesia, or neurological conditions. Diagnosis of rTTC requires the presence of LV basal hypokinesis/akinesis, new electrocardiogram (EKG) abnormalities or elevated cardiac troponin, and absence of obstructive coronary disease, pheochromocytoma, or myocarditis. Management of rTTC is similar to that of TTC, which is predominantly supportive with the treatment of complications. The recurrence rate of rTTC is around $10 \%$. The most common complications of rTTC include myocarditis, pleural and pericardial effusions, and development of LV thrombi. The best predictors of mortality include decreased LVEF, development of atrial fibrillation, and neurologic disease.
\end{abstract}

Keywords: Cardiomyopathy; Takotsubo; reverse-Takotsubo; stress; heart failure

Submitted Oct 16, 2018. Accepted for publication Nov 01, 2018.

doi: $10.21037 /$ atm.2018.11.08

View this article at: http://dx.doi.org/10.21037/atm.2018.11.08

\section{Introduction}

\section{History of Takotsubo cardiomyopathy (TTC)}

TTC, also known as "stress cardiomyopathy" or "broken heart syndrome," was first described in 1983 in Japanese females (1). It predominantly occurs in the postmenopausal women and is usually triggered by emotional or physical stress. It is an acute but often reversible left ventricular
(LV) dysfunction. TTC has a clinical presentation often resembling acute coronary syndrome (ACS) with no evident obstructive coronary artery disease on angiogram. It is characterized by a transient cardiac apical akinesis/ hypokinesis and basal hyperkinesis that resolves spontaneously within a few days or weeks (2). TTC received its name due to the characteristic apical ballooning of the heart giving it a shape similar to a "Takotsubo", a Japanese 
Table 1 Pathophysiological mechanisms of reverse takotsubo cardiomyopathy

\begin{tabular}{ll}
\hline Proposed pathophysiological mechanisms of reverse takotsubo cardiomyopathy & Justification for proposed mechanism \\
\hline Catecholamine cardiotoxicity & Stress, fear and excitement \\
Coronary artery spasm & Transient ischemia, similar presentation to ACS \\
Coronary microvasculature impairment & Ischemia, similar presentation to ACS \\
Estrogen deficiency & Postmenopausal women \\
\hline
\end{tabular}

ACS, acute coronary syndrome.

pot used to trap an octopus. Several variants of TTC have been described based on the regions of the ventricular wall motion abnormality including the midventricular and basal wall. Reverse Takotsubo cardiomyopathy (rTTC) is a rare variant of TTC which presents with basal ballooning instead of apical ballooning (3). This manuscript aims to perform a comprehensive literature review of epidemiology, pathophysiology, clinical presentation, diagnosis, and treatment of rTTC, and highlight differences with TTC when present.

\section{Epidemiology}

The incidence of TTC is estimated to be around $2 \%$ of all troponin-positive patients presenting with suspected ACS $(4,5)$. However, the reported proportion with the rTTC variant out of all TTC patients in published literature has been variable, ranging from $1-23 \%$ (6-8). In a literature review of case reports, the proportion of rTTC in 60 TTC cases was $23.3 \%$ (7). In a more extensive study that analyzed records of 1,750 patients from the International Takotsubo Registry showed that only $2.2 \%$ of patients had the reversed variant (6). Only $10 \%$ of all cases of TTC have been reported in men; the majority of cases are women of postmenopausal age, 65-70 years old (9). While TTC typically presents in postmenopausal women, rTTC has been observed mostly in younger women.

\section{Pathophysiology}

While the exact mechanism of development of this cardiomyopathy is unknown, various theories such as catecholamine cardiotoxicity, coronary artery spasm, coronary microvascular impairment, and estrogen deficiency have been proposed (Table 1).

\section{Catecholamine cardiotoxicity}

One of the most popular theories for the pathophysiological mechanism in the development of rTTC, and TTC, in general, is the catecholamine-induced cardiotoxicity. In vivo studies in animal models have shown the development of acute reversible apical hypokinesia of the heart after the administration of intraperitoneal or intravenous catecholamines. This is often associated with increased systolic and diastolic blood pressures. Histologically, catecholamine cardiotoxicity in animals presents with myocardial contraction band necrosis, neutrophil infiltration, and fibrosis. The reversible apical hypokinesia has also been induced in rats by exposure to emotional stressors including immobilization possibly due to a regional heterogeneity of catecholamine sensitivity (10). Another suggested mechanism is that epinephrine or norepinephrine bind to B2 receptors on myocardial tissue triggering intracellular signaling from Gs protein to Gi protein coupling which in turn activates protein kinase A via cyclic AMP and produces an increased contractile response (11). Increased cyclic AMP triggers the formation of free radicals initiating the expression of stress response genes and induces apoptosis (12). A retrospective study from Japan on 328 patients with TTC variants and subarachnoid hemorrhage, reported that the patients with rTTC exhibited significantly higher levels of plasma epinephrine in comparison to the patients with TTC. No significant differences were detected in plasma norepinephrine levels (13) (Figure 1).

\section{Coronary artery spasm}

The reversible ventricular dysfunction of rTTC is also theorized to be caused by a coronary artery spasm resulting 


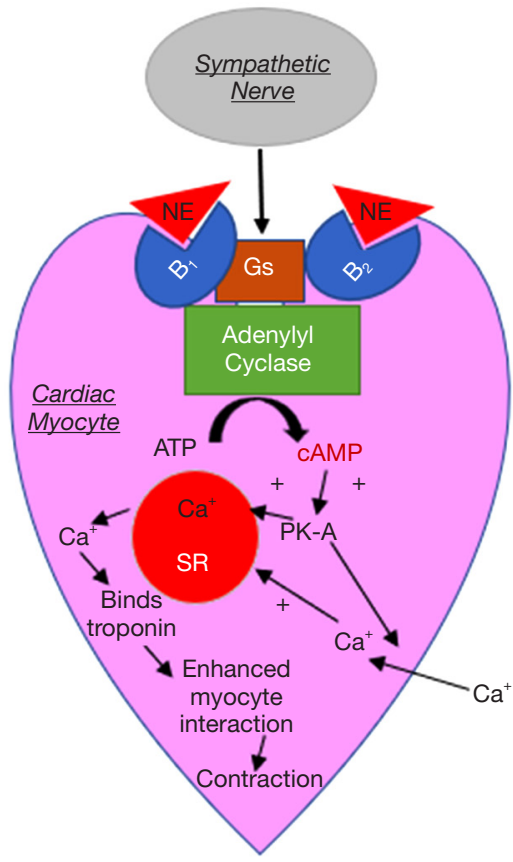

Figure 1 Proposed mechanism of catecholamine cardiotoxicity. $\mathrm{Ne}$, norepinephrine; Gs, G-stimulatory protein; PK-A, cAMPdependent protein kinase; SR, sarcoplasmic reticulum.

from improper coronary blood flow from a vulnerable plaque rupture. However, it has been argued that ischemic myocardial stunning does not typically produce the histological changes observed in TTC. According to a literature review of 272 TTC case reports, spontaneous coronary arterial spasm was suspected in $1 \%$, but it was never actually observed (14).

\section{Coronary microvascular impairment}

Disturbances in the coronary microcirculation have also been proposed as a mechanism for the development of rTTC due to abnormal $\mathrm{LV}$ wall motion in the large areas of the myocardium which is dynamic rather than fixed (14). The various disturbances include impaired endotheliumdependent vasodilation, excessive vasoconstriction, and reduced myocardial perfusion (11).

\section{Estrogen deficiency}

Since around $90 \%$ of TTC patients are postmenopausal women, estrogen deficiency has been suggested as an underlying mechanism (11). Estrogen withdrawal has been associated with the impaired coronary microvascular function. In oophorectomized rats, estrogen replacement diminished inhibitory effects on myocardial contraction induced by high levels of epinephrine. This is suspected to be mediated by an increased activity of the B2 adrenoceptor signaling pathway and decreasing concentration of catecholamines in the plasma. The estrogen receptors which are expressed on the cardiac myocytes can modulate cardiac contractility and regulate calcium uptake (10).

\section{Triggers of rTTC}

While up to $30 \%$ of all patients with TTC have no clearly identified triggers, a variety of stimuli can precipitate the condition. Emotional and physical stress is the most commonly recognized triggers $(15,16)$. rTTC has also been reported in the patients with various types of life-threatening intracranial hemorrhages including subarachnoid and medullary hemorrhages $(13,17,18)$. Patients have also developed rTTC while under general anesthesia for surgical and dental procedures (19-23). It is not clear if this occurrence is directly related to the use of anesthetic agents or the emotional stress associated with the procedure. Eating disorders and malnutrition have also been correlated with the occurrence of rTTC (24). Development of rTTC has also been associated with neurologic conditions, such as multiple sclerosis and serotonin syndrome $(25,26)$. Additionally, patients have presented with rTTC after administration of exogenous catecholamines which supports the theory of catecholamine-induced myocardial stunning (27-29). The use of amphetamine and methamphetamine has also been recognized as a trigger for rTTC $(30,31)$. Some of the infrequent triggers of rTTC include black widow spider bites, use of the drug yohimbine and consumption of energy drinks (32-34).

\section{Diagnosis of rTTC}

TTC often symptomatically mimics ACS or ischemic heart disease. Similarly, rTTC often presents with chest pain, dyspnea, nausea, abdominal pain, diaphoresis, epigastric pain, syncope, or indigestion (35). Electrocardiography changes ( $\mathrm{T}$ wave inversion, ST-segment elevation, prolonged QT interval, new bundle branch block) and LV wall motion abnormalities (hypokinesis or dyskinesis of LV apex) are often limited to one coronary artery territory. Cardiac troponin level is typically elevated. The typical diagnostic workup, usually due to suspected ACS, includes 
an electrocardiogram (EKG), blood sample analysis, transthoracic echocardiogram, and coronary angiogram. In addition, ventriculogram and cardiac magnetic resonance imaging (MRI) are sometimes performed. The ultimate diagnosis is typically made with cardiac catheterization during left ventriculography (35).

\section{Clinical presentation of $r T T C$}

Similar to TTC, the patients with rTTC present with chest pain and/or dyspnea preceded by an emotional or physically stressful event with angina-like chest pain. Additional symptoms of rTTC patients include syncope, nausea, abdominal pain, diaphoresis, epigastric pain, and indigestion. These patients also frequently present with mild to moderate congestive heart failure represented by a decreased ejection fraction. They can have hypotension due to reduced stroke volume or $\mathrm{LV}$ outflow obstruction. In a study of 1,750 patients from the International Takotsubo Registry, patients with rTTC presented more often with a neurological disorder while patients with TTC presented with an acute psychiatric episode (36). rTTC is typically more likely to be complicated by pulmonary edema and cardiogenic shock $(13,16,37)$.

\section{Diagnostic work-up of rTTC}

Diagnostic criteria are shared between TTC and rTTC with the difference being in the region of the myocardium affected. Mayo clinic published the revised rTTC diagnosis criteria in $2008(38,39)$. These criteria define rTTC as an acute event characterized by all the following: (I) transient abnormal wall-motion in the form of hypokinesis, akinesis, or dyskinesis of the LV basal segments, with the regional affected extending beyond a single epicardial vascular distribution; (II) no detectable obstructive coronary disease or angiographic evidence of acute atheromatous plaque rupture; (III) newly developed ECG abnormalities or elevation in cardiac troponin levels; and (IV) absence of myocarditis or pheochromocytoma (38).

To support these criteria, Eitel et al. performed a prospective study on 256 TTC patients at seven tertiary care centers in Europe and North America over a 5-year period. All patients underwent cardiac catheterization, and unobstructed coronary arteries were seen in about $75 \%$ of patients (8).

\section{EKG abnormalities of rTTC}

The rTTC and TTC patients often present with EKG abnormalities. The most common reported EKG abnormality is ST-segment elevations mimicking STelevation myocardial infarction (STEMI) presentation (40). However, significant variation in the occurrence of this finding is reported in the published literature which could be due to several factors: the transient elevations and selection bias for STEMI to receive immediate coronary angiography. The ST segment elevation is usually seen in the precordial leads, but it can also be seen in the inferior or lateral leads as well which typically resolves within 2 to 3 days after the event. Other types of ECG abnormalities seen in rTTC include $\mathrm{T}$ wave inversion, new bundle branch blocks, and prolonged QT interval. T wave inversion and QT prolongation on average resolves over 3 to 4 months (39). In the International Takotsubo Registry study, patients with typical TTC presented more often with ST-segment elevation, atrial fibrillation, and T-wave inversion. Patients with rTTC presented more frequently with ST-segment depression and longer QTc duration (36).

\section{Laboratory analysis}

Most patients with rTTC present with a modest rise in cardiac troponin levels that peak within 24 hours. Circulating brain natriuretic peptide (BNP) is also typically elevated as a marker of ventricular dysfunction and is correlated with LV end-diastolic pressure (39). In the International Takotsubo Registry, upon admission, TTC patients were found to have higher levels of BNP and c-reactive protein than patients with rTTC (36).

\section{Coronary angiogram}

Coronary angiograms can detect atherosclerosis and cardiac wall motion abnormalities. In rTTC patients, the coronary arteries are typically normal or have mild atherosclerosis. In rTTC patients, wall motion abnormality includes hypokinesis/akinesis of the basal segment of the left ventricle. While in TTC, the wall motion abnormality is hypokinesis or akinesis of the apical and mid segments of the left ventricle with sparing of basal systolic function. Right ventricle can have similar motion abnormality in patients who tend to be sicker and at higher risk of 
developing congestive heart failure (39).

\section{Cardiac MRI}

The use of Cardiac MRI in the diagnosis of rTTC quantifies $\mathrm{LV}$ and right ventricular (RV) function, gives an accurate visualization of regional wall motion abnormalities, and possible abnormalities/complications (i.e., pericardial effusion, pleural effusion, LV and RV thrombi). Cardiac MRI differentiates reversible injury (inflammation, ischemic edema) and irreversible injury (necrosis, fibrosis), using delayed gadolinium hyperenhancement, to exclude the differential diagnoses of myocardial infarction and myocarditis $(8,39,41)$.

\section{Ejection fraction}

On average, TTC patients appear to present with lower left ventricular ejection fraction (LVEF) in comparison to rTTC patients. In a systematic review of 136 TTC patients, Sharkey et al. analyzed the normalization of LVEF over time with a transthoracic echocardiogram. Upon admission, the average LVEF for patients was found to range from $15 \%$ to $55 \%$. One hundred thirty-one patients followed up (in the hospital after three days or after discharge at 51 to 52 days) to monitor the LVEF; 126 of these patients eventually returned to normal or greater than or equal to $50 \%$. Only six patients took 2.5 to 12 months until their LVEF normalized (42). In the International Takotsubo Registry study, upon admission, patients with TTC had a lower LVEF (mean, $41 \%$ ) than rTTC patients (mean, 43\%) (36).

\section{Management of rTTC}

According to the American College of Cardiology (ACC) and the European Society of Cardiology, management of rTTC is predominantly supportive with the management of complications $(35,43)$. The treatment guidelines for rTTC are similar to those of TTC except for circumstances when the reversed anatomy is significant. Immediate aggressive pharmacologic and hemodynamic supports rapidly reversed LV function and survival. Due to an initial presentation that mimics ACS, patients are generally acutely treated according to the guidelines or ACS management.

For rTTC patients with dynamic LV obstruction, beta blockers can be cautiously given to reduce the contractility of the myocardial segment affected. In this case, inotropes are contraindicated (35). If the patient presents with low blood pressure secondary to cardiogenic shock, vasopressor agents, including dobutamine and dopamine, are indicated; intra-aortic balloon counterpulsation may also be needed for mechanical and hemodynamic support for 1 to 2 days (44). Intracavity thrombus formation can occur due to the akinetic ventricular segments. Suspicion of thrombus formation in the akinetic basal segment should be treated with prophylactic anticoagulation, such as warfarin, for at least three months regardless of the heart rhythm (5). In patients with symptomatic hypotension without outflow tract obstruction, catecholamines can be given (35). Pulmonary edema is treated with upright posture, oxygen, and diuretics; however, sedation and morphine can also be used (45).

While arrhythmia from QT prolongation is common in rTTC, antiarrhythmics are generally not given prophylactically. Magnesium sulfate can be given for ventricular tachycardia with prolonged QT interval in the acute phase of rTTC.

Multiple new treatments for management of TTC and rTTC are under investigation. In an animal model, estrogen treatment has been beneficial in preventing the condition, however, clinical trials of the administration of estrogen in patients have not been performed (14). The role of the routine use of adenosine and endothelin antagonist for management of TTC in general needs to be further investigated. Long-term therapy with beta-blockers has been hypothesized to reduce the likelihood of recurrence. However, more research needs to be done to support this theory (5).

\section{Prognosis of rTTC}

The recurrence rate and outcomes of rTTC have been found to be very similar to those of TTC (36). In a study of 100 TTC patients, over a 4-year follow up, TTC recurred in a total $10 \%$ of patients. This study estimates a $2.9 \%$ recurrence rate in the first 4 years after the initial event and a recurrence rate of $1.3 \%$ (46) per year for subsequent follow ups. The most common complications associated with rTTC include myocardial inflammation, pleural effusion, pericardial effusion, and LV thrombi. No significant difference in the mortality was found between the TTC patients and the controls (46). The best predictors of 1-year mortality were unrelated to the variant of TTC. Instead, LVEF less than $45 \%$, atrial fibrillation, and neurologic disease were significantly associated with 
increased risk of 1-year mortality (36).

\section{Acknowledgements}

None.

\section{Footnote}

Conflicts of Interest: The authors have no conflicts of interest to declare.

\section{References}

1. Aizawa K, Suzuki T. Takotsubo Cardiomyopathy. Japanese Perspective. Heart Fail Clin 2013;9:243-7.

2. Dote K, Sato H, Tateishi H, et al. Myocardial stunning due to simultaneous multivessel coronary spasms: a review of 5 cases. J Cardiol 1991;21:203-14.

3. Sharkey SW, Maron BJ. Epidemiology and Clinical Profile of Takotsubo Cardiomyopathy. Circ J 2014;78:2119-28.

4. Kurowski V, Kaiser A, von Hof K, et al. Apical and midventricular transient left ventricular dysfunction syndrome (tako-tsubo cardiomyopathy): frequency, mechanisms, and prognosis. Chest 2007;132:809-16.

5. Sharkey SW, Windenburg DC, Lesser JR, et al. Natural History and Expansive Clinical Profile of Stress (Tako-Tsubo) Cardiomyopathy. J Am Coll Cardiol 2010;55:333-41.

6. Ghadri JR, Cammann VL, Napp LC, et al. Differences in the Clinical Profile and Outcomes of Typical and Atypical Takotsubo Syndrome Data From the International Takotsubo Registry. JAMA Cardiol 2016;1:335-40.

7. Ramaraj R, Movahed MR. Reverse or Inverted Takotsubo Cardiomyopathy (Reverse Left Ventricular Apical Ballooning Syndrome) Presents at a Younger Age Compared With the Mid or Apical Variant and Is Always Associated With Triggering Stress. Congest Heart Fail 2010;16:284.

8. Eitel I, von Knobelsdorff-Brenkenhoff F, Bernhardt P, et al. Clinical characteristics and cardiovascular magnetic resonance findings in stress (takotsubo) cardiomyopathy. JAMA 2011;306:277-86.

9. Kurisu S, Kihara Y. Tako-tsubo cardiomyopathy: Clinical presentation and underlying mechanism. J Cardiol 2012;60:429-37.

10. Williams R, Arri S, Prasad A. Current Concepts in the Pathogenesis of Takotsubo Syndrome. Heart Fail Clin 2016;12:473-84.
11. Milinis K, Fisher M. Takotsubo cardiomyopathy: Pathophysiology and treatment. Postgrad Med J 2012;88:530-8.

12. Litvinov I V, Kotowycz MA, Wassmann S. Iatrogenic epinephrine-induced reverse Takotsubo cardiomyopathy: direct evidence supporting the role of catecholamines in the pathophysiology of the "broken heart syndrome". Clin Res Cardiol 2009;98:457-62.

13. Kumai T, Inamasu J, Watanabe E, et al. Differences between Takotsubo cardiomyopathy and reverse Takotsubo cardiomyopathy associated with subarachnoid hemorrhage. Int J Cardiol Heart Vasc 2016;11:99-103.

14. Akashi YJ, Goldstein DS, Barbaro G, et al. Takotsubo cardiomyopathy: a new form of acute, reversible heart failure. Circulation 2008;118:2754-62.

15. Templin C, Ghadri JR, Diekmann J, et al. Clinical Features and Outcomes of Takotsubo (Stress) Cardiomyopathy. N Engl J Med 2015;373:929-38.

16. Barbaryan A, Bailuc SL, Patel K, et al. An Emotional Stress as a Trigger for Reverse Takotsubo Cardiomyopathy: A Case Report and Literature Review. Am J Case Rep 2016;17:137-42.

17. Gobeske KT, Sarano ME, Fugate JE, et al. Medulla Oblongata Hemorrhage and Reverse Takotsubo Cardiomyopathy. Neurocrit Care 2017. [Epub ahead of print].

18. Elikowski W, Małek-Elikowska M, Kudliński B, et al. ECG pattern in reverse takotsubo cardiomyopathy demonstrated in 5 cases with intracranial hemorrhage. Pol Merkur Lekarski 2016;41:136-40.

19. Lee CH, Son JW, Kim U. Reverse Takotsubo Cardiomyopathy following Inadvertent Intrathecal Injection during Percutaneous Epidural Neuroplasty. Heart Lung Circ 2015;24:e148-51.

20. Bleser T, Weth C, Görge G. Reverse takotsubo cardiomyopathy-a life-threatening disease. Successful resuscitation of a 31-year-old woman with cardiologic shock after a visit to the dentist. Med Klin Intensivmed Notfmed 2013;108:675-8.

21. Khalili A, Dabbous A, Taha S, et al. Reverse Takotsubo Cardiomyopathy During General Anesthesia in a 16-YearOld Female Victim of War. J Cardiothorac Vasc Anesth 2018;32:1858-62.

22. Demir GG, Babur Güler G, Güler E, et al. Sinus surgery complicated by ventricular fibrillation in a young patient: Inverted (reverse) Takotsubo cardiomyopathy. Turk Kardiyol Dern Ars 2016;44:418-22.

23. Açar B, Kırbaş Ö, Ünal S, et al. Reverse Takotsubo 
cardiomyopathy following intra-abdominal surgery. Turk Kardiyol Dern Ars 2016;44:514-6.

24. Tagami T, Mertens A, Rothschild D, et al. Case Report: A case of reverse takotsubo cardiomyopathy caused by an eating disorder. J Cardiol Cases 2016;15:77-9.

25. Mehta NK, Aurigemma G, Rafeq Z, et al. Reverse Takotsubo Cardiomyopathy after an Episode of Serotonin Syndrome. Tex Heart Inst J 2011;38:568-72.

26. Peller M, Balsam P, Budnik M, et al. Reverse Takotsubo syndrome in a patient with diagnosed multiple sclerosis. Kardiol Pol 2016;74:1029.

27. Esnault P, Née L, Signouret T, et al. Reverse Takotsubo cardiomyopathy after iatrogenic epinephrine injection requiring percutaneous extracorporeal membrane oxygenation. Can J Anaesth 2014;61:1093-7.

28. Belliveau D, De S. Reverse Takotsubo Cardiomyopathy following Exogenous Epinephrine Administration in the Early Postpartum Period. Echocardiography 2016;33:1089-91.

29. Khoueiry G, Abi Rafeh N, Azab B, et al. Clinical Communications: Adults: Reverse Takotsubo Cardiomyopathy in the Setting of Anaphylaxis Treated with High-dose Intravenous Epinephrine. J Emerg Med 2013;44:96-9.

30. Movahed MR, Mostafizi K. Reverse or inverted left ventricular apical ballooning syndrome (reverse Takotsubo cardiomyopathy) in a young woman in the setting of amphetamine use. Echocardiography 2008;25:429-32.

31. Chehab O, Ioannou A, Sawhney A, et al. Clinical Communications: Adult: Reverse Takotsubo Cardiomyopathy and Cardiogenic Shock Associated With Methamphetamine Consumption. J Emerg Med 2017;53:e81-3.

32. Alexakis L-C, Arapi S, Stefanou I, et al. Transient reverse takotsubo cardiomyopathy following a spider bite in Greece: a case report. Medicine (Baltimore) 2015;94:e457.

33. Rodriguez-Castro CE, Saifuddin F, Porres-Aguilar M, et al. Reverse takotsubo cardiomyopathy with use of male enhancers. Proc (Bayl Univ Med Cent) 2015;28:78-80.

34. Kaoukis A, Panagopoulou V, Mojibian HR, et al. Reverse Takotsubo Cardiomyopathy Associated With the Consumption of an Energy Drink. Circulation 2012;125:1584-5.

Cite this article as: Awad HH, McNeal AR, Goyal H. Reverse Takotsubo cardiomyopathy: a comprehensive review. Ann Transl Med 2018;6(23):460. doi: 10.21037/atm.2018.11.08
35. Amsterdam EA, Wenger NK, Brindis RG, et al 2014 AHA/ACC Guideline for the Management of Patients With Non-ST-Elevation Acute Coronary Syndromes. Circulation 2014;130:e344-426.

36. Ghadri JR, Cammann VL, Napp LC, et al. Differences in the clinical profile and outcomes of typical and atypical takotsubo syndrome: Data from the international takotsubo registry. JAMA Cardiol 2016;1:335-40.

37. Ikram S, Saleem N, Latif RK. Acute left ventricle failure on induction of anesthesia: a case report of reverse stress cardiomyopathy - presentation, diagnosis and treatment. J Anesth 2016;30:911-4.

38. Scantlebury DC, Prasad A. Diagnosis of Takotsubo Cardiomyopathy - Mayo Clinic Criteria. Circulation 2014;78:2129-39.

39. Prasad A, Lerman A, Rihal CS. Curriculum in Cardiology: Apical ballooning syndrome (Tako-Tsubo or stress cardiomyopathy): A mimic of acute myocardial infarction. Am Heart J 2008;155:408-17.

40. Bybee KA, Prasad A. Stress-Related Cardiomyopathy Syndromes. Circulation 2008;118:397-409.

41. Eitel I, Behrendt F, Schindler K, et al. Differential diagnosis of suspected apical ballooning syndrome using contrast-enhanced magnetic resonance imaging. Eur Heart J 2008;29:2651-9.

42. Sharkey SW, Windenburg DC, Lesser JR, et al. Quarterly Focus Issue: Heart Failure: Natural History and Expansive Clinical Profile of Stress (Tako-Tsubo) Cardiomyopathy. J Am Coll Cardiol 2010;55:333-41.

43. Lyon AR, Bossone E, Schneider B, et al. Current state of knowledge on Takotsubo syndrome: a Position Statement from the Taskforce on Takotsubo Syndrome of the Heart Failure Association of the European Society of Cardiology. Eur J Heart Fail 2016;18:8-27.

44. Sharkey SW, Lesser JR, Zenovich AG, et al. Acute and Reversible Cardiomyopathy Provoked by Stress in Women From the United States. Circulation 2005;111:472-9.

45. Akashi YJ, Goldstein DS, Barbaro G, et al. Takotsubo Cardiomyopathy. Circulation 2008;118:2754-62.

46. Elesber AA, Prasad A, Lennon RJ, et al. Four-Year Recurrence Rate and Prognosis of the Apical Ballooning Syndrome. J Am Coll Cardiol 2007;50:448-52. 